\title{
Análise da lateralidade e Destreza Manual em Crianças com Transtorno do Espectro Autista ${ }^{1}$ \\ ANALYSIS OF LATERALITY AND MANUAL DEXTERITY IN CHILDREN WITH AUTISTIC SPECTRUM DISORDER
}

\author{
Lidiane Aparecida FERNANDES ${ }^{2}$ \\ Andressa SILVA ${ }^{3}$ \\ Violeta Marina Echevarria AUGUSTO ${ }^{4}$ \\ Nathálya Gardênia de Holanda Marinho NOGUEIRA ${ }^{5}$ \\ Bárbara de Paula FERREIRA ${ }^{6}$ \\ Cristiani JUNQUEIRA ${ }^{7}$ \\ Guilherme Menezes LAGE ${ }^{8}$
}

\begin{abstract}
RESUMO: Este estudo teve como objetivo analisar a lateralidade e a destreza manual em crianças com Transtorno do Espectro Autista (TEA). Do ponto de vista da motricidade humana, a lateralidade contribui para o processo de maturação motora. Figurase entre as principais características do autismo o atraso nas habilidades motoras grossas e finas, com piora progressiva no quadro. Analisar o nível de lateralidade do indivíduo com TEA torna-se importante por possibilitar uma direção na intervenção motora com o intuito de melhorar a funcionalidade do autista e sua qualidade de vida. A amostra deste estudo foi composta por oito crianças, alunos de uma instituição de ensino pública, do sexo masculino, com idade média de 8,75 1,83 anos e diagnosticadas com TEA com base no DSM-V. Os resultados mostraram que os participantes do estudo parecem apresentar lateralidade destra e, também, melhor desempenho com a mão preferida em uma tarefa de destreza manual. Observou-se que, na tarefa que exigiu maior atenção e destreza, a diferença no desempenho das mãos foi significativa. Já na tarefa com menor demanda atencional e de destreza manual, o desempenho entre as mãos não foi significante, porém foi nesse momento que foram observados os maiores erros na execução. A motivação pode ser uma variável fundamental para o desempenho motor em tarefas que avaliam o tempo de execução. É importante uma maior ênfase no desenvolvimento da motricidade desses indivíduos durante a fase escolar para diminuir as dificuldades motoras e, consequentemente, melhorar a qualidade de vida, sensação de bem-estar, autonomia e interação social.
\end{abstract}

PALAVRAS-CHAVE: Lateralidade. Coordenação viso-motora. Autismo.

ABSTRACT: This study aimed to analyze laterality and manual dexterity in children with Autism Spectrum Disorder (ASD). From the point of view of human motor skills, laterality contributes to the motor maturation process. Among the main characteristics of autism is the delay in gross and fine motor skills, with progressive worsening in the condition. Analyzing the level of laterality of the individual with ASD becomes important because it allows a direction in the motor intervention in order to improve the functionality and quality of life of the autistic person. The sample of this study was composed of eight children, students of a public education institution, male, with an average age of $8.75 \pm 1.83$ years old and diagnosed with ASD based on the DSM-V.

\footnotetext{
${ }^{1}$ https://doi.org/10.1590/1980-54702020v26e0084

${ }^{2}$ Universidade Federal de Minas Gerais (UFMG). Escola de Educação Física, Fisioterapia e Terapia Ocupacional (EEFFTO). Belo Horizonte/Minas Gerais/Brasil. E-mail: lidianefernandes12@yahoo.com.br. ORCID: https://orcid.org/0000-0001-8909-1612

${ }^{3}$ Universidade Federal de Minas Gerais (UFMG). Escola de Educação Física, Fisioterapia e Terapia Ocupacional (EEFFTO). Belo Horizonte/Minas Gerais/Brasil. E-mail: silvadressa@gmail.com. ORCID: https://orcid.org/0000-0002-0408-8631

${ }^{4}$ Universidade Federal de Minas Gerais (UFMG). Escola de Educação Física, Fisioterapia e Terapia Ocupacional (EEFFTO). Belo Horizonte/Minas Gerais/Brasil. E-mail: violeta.eaugusto@gmail.com. ORCID: https://orcid.org/0000-0001-5977-915X

${ }^{5}$ Universidade Federal de Minas Gerais (UFMG). Escola de Educação Física, Fisioterapia e Terapia Ocupacional (EEFFTO). Belo Horizonte/Minas Gerais/Brasil. E-mail: marinhohnathy@gmail.com. ORCID: https://orcid.org/0000-0003-0286-7559

${ }^{6}$ Universidade Federal de Minas Gerais (UFMG). Escola de Educação Física, Fisioterapia e Terapia Ocupacional (EEFFTO). Belo Horizonte/Minas Gerais/Brasil. E-mail: barbaradepaulaf@gmail.com. ORCID: https://orcid.org/0000-0001-8347-0558

${ }^{7}$ Universidade Federal de Minas Gerais (UFMG). Escola de Educação Física, Fisioterapia e Terapia Ocupacional (EEFFTO). Belo Horizonte/Minas Gerais/Brasil. E-mail: cristianijunqueira74@gmail.com. ORCID: https://orcid.org/0000-0003-1081-8984

${ }^{8}$ Universidade Federal de Minas Gerais (UFMG). Escola de Educação Física, Fisioterapia e Terapia Ocupacional (EEFFTO). Belo Horizonte/Minas Gerais/Brasil. E-mail: menezeslage@gmail.com. ORCID: https://orcid.org/0000-0001-8296-3676
} 
The results showed that the study participants seem to have right handedness and also better performance with the preferred hand in a manual dexterity task. It was observed that, in the task that demanded more attention and dexterity, the difference in hand performance was significant. In the task with the lowest attentional demand and manual dexterity, the performance between the hands was not significant; however, it was at that moment that the biggest mistakes in execution were observed. Motivation can be a fundamental variable for motor performance in tasks that assess execution time. It is important to emphasize the development of the motor skills of these individuals during the school phase to reduce motor difficulties and, consequently, improve quality of life, sense of well-being, autonomy and social interaction.

KEYWORDS: Laterality. Visual-motor coordination. Autism.

\section{INTRODUÇÃo}

O Transtorno do Espectro Autista (TEA) é um distúrbio do neurodesenvolvimento que se caracteriza por alteraçóes presentes desde idade muito precoce, tipicamente antes dos três anos de idade, com comprometimento em três áreas: interação social, comunicação e comportamento restrito e repetitivo (Caetano, 1993; Catelli, D’Antino, \& Assis 2016; Günal, Bumin, \& Huri 2019). Devido à grande variação em grau e intensidade de suas manifestaçóes, o Manual de Diagnóstico e Estatístico de Transtornos Mentais 5a edição - DSM-V (American Psychiatric Association [APA], 2013), categorizou todas as apresentaçôes fenotípicas do autismo como TEA, o qual inclui transtorno autístico (autismo), síndrome de Asperger, transtorno desintegrativo da infância e transtorno global ou invasivo do desenvolvimento sem outra especificação (Vitorino, 2014).

Historicamente, a primeira vez que o TEA foi relatado em estudos científicos foi em 1943 pelo Dr. Leo Kanner, quando observou 11 crianças que apresentavam comportamentos peculiares de interação social, comunicação e estereotipias (Chukoskie, Townsend, \& Westerfield, 2013; Pereira, Riesgo, \& Wagner, 2008). Ao extrapolar as causas do distúrbio, Pereira, Riesgo e Wagner (2008) relacionou o afastamento social das crianças com o caráter altamente intelectual dos pais, dando uma condição psicossocial à doença. Entretanto, essa relação foi praticamente descartada, uma vez que trabalhos subsequentes esclareceram que o TEA é um distúrbio cerebral com um forte componente genético (Rutter \& Thapar, 2014).

A partir de então, as convicçóes do fator neurobiológico para a etiologia do TEA ganharam força e as pesquisas começaram a ser mais direcionadas para as bases cerebrais do transtorno, sugerindo que algumas regióes cerebrais estariam alteradas. Embora as principais características do TEA sejam o isolamento social, falta de contato visual e déficits na capacidade de linguagem, as funções motoras tipicamente são alteradas (Kummer \& Teixeira, 2007). Autores que avaliaram habilidades motoras em autistas, como Lloyd, MacDonald e Lord (2013), afirmam que habilidades motoras grossas e finas de crianças com TEA são atrasadas e se tornam progressivamente mais atrasadas com a idade.

Uma das possíveis explicaçóes para o atraso motor encontrado no TEA pode estar relacionada à variabilidade da especialização hemisférica dessa população, estando o hemisfério direito mais desenvolvido do que o esquerdo, alterando algumas capacidades, tais como motoras, auditivas e visuais (Molfese \& Segalowitz, 1988; Zilbovicius, Meresse, \& Boddaert, 2006). A especialização hemisférica é uma explicação biológica para o desenvolvimento da lateralidade. Além disso, a diferença no desenvolvimento dos hemisférios cerebrais (Molfese \& Segalowitz, 1988) pode resultar em diferentes padrōes de desenvolvimento da lateralidade. 
Em decorrência da grande variabilidade da especialização hemisférica encontrada no TEA, poucos estudos sobre essa temática são encontrados na literatura (Aram et al., 1988; Floris \& Henrietta, 2018; Paquet, Golse, Girard, Olliac, \& Vaivre-Douret, 2017; Zilbovicius, Meresse, \& Boddaert, 2006). A lateralidade como elemento da motricidade humana está diretamente relacionada à precisão, à preferência, à velocidade e à coordenação do movimento, contribuindo no processo de maturação motora (Velasco, 1996). O estabelecimento tardio da lateralidade em crianças com TEA pode ter um impacto significativo no desenvolvimento de certas aquisiçóes, como a escrita, a coordenação visual-motora, a destreza manual e a linguagem (Paquet et al., 2017). Nesse aspecto, quanto mais precoce o desenvolvimento da lateralidade, que nos autistas é menos desenvolvida em comparação a de grupos típicos (Thompson et al., 2017), melhor será o desenvolvimento da criança (Hauck \& Dewey, 2001; Romero, 1988).

Essa dimensão do movimento humano, a lateralidade, é essencial para o desenvolvimento global da criança, uma vez que interfere diretamente na forma como ela irá socializar com o mundo. De acordo com Hauck e Dewey (2001), as crianças autistas que são mais lateralizadas manualmente possuem melhores desempenho motor, verbal e cognitivo em comparação às menos lateralizadas. A lateralidade impacta nas interaçóes sociais de crianças com TEA, tais como na forma como arremessa uma bola ou abre uma porta utilizando uma chave (Paquet et al., 2017). Além disso, a lateralidade manual no TEA impacta no desenvolvimento da aquisição da escrita, destreza manual e linguagem e pode atuar como marcador comportamental que diz respeito ao funcionamento cerebral da criança com TEA, estando associado a menor capacidade cognitiva (Forrester, Ruth, Thomas, \& Mareschal 2017). Como exemplo, crianças com TEA que apresentam lateralidade mais definida tende a ter capacidade linguística mais desenvolvida do que crianças menos lateralizadas (Rodriguez et al., 2010). A partir disso, saber o nível de lateralidade do indivíduo com TEA torna-se importante por ter implicaçóes para futuros diagnósticos e por possibilitar uma direção na intervenção motora com o intuito de melhorar a funcionalidade dos autistas e, consequentemente, sua qualidade de vida (Preslar, Kushner, Marino, \& Pearce 2013).

Avaliar a lateralidade parece, à primeira vista, uma tarefa muito simples, porém os resultados podem variar substancialmente, principalmente em indivíduos com TEA, em decorrência das suas dificuldades motoras e da variação dos níveis de comprometimento. Os estudos que estabelecem os resultados padróes para esse grupo são escassos e possuem restriçóes no seu poder de generalização. Além disso, vale enfatizar a importância da utilização de testes adequados, pois são úteis na identificação de alteraçóes nos padróes motores, principalmente na fase escolar (Rosa Neto, 2002), auxiliando o direcionamento de intervençóes específicas para cada autista na tentativa de melhorar sua qualidade de vida por meio do movimento.

A avaliação da preferência lateral pode ser realizada a partir de alguns instrumentos, como exemplo: questionários, a realização de praxias usuais e a execução de tarefas que requeiram destreza manual. Nos estudos de revisão de literatura conduzidos por Catelli, D’Antino, \& Assis (2016) e Soares e Cavalcante Neto (2015), foi realizado um mapeamento dos instrumentos de avaliação motora em individuos com TEA. Em ambos os estudos, foi possível concluir que há uma escassez de literatura relacionada a esse tema, principalmente no Brasil. No que se refere à credibilidade dos instrumentos encontrados, os autores questionam se realmente são adequados para a população com TEA. 
Neste estudo, optamos pela utilização do Nine Hole Pegboard Test (9-HPT), pois é um teste de administração fácil e rápida. Além disso, funciona como uma medida confiável e válida de destreza manual (Poole et al., 2005; Smith, Hong, \& Presson 2000). Em diversas populaçóes, foi demonstrada a capacidade de avaliação da destreza manual utilizando o 9-HPT, como, por exemplo, acidente vascular encefálico, encefalopatia crônica não progressiva da infância, comprometimento cerebelar, doença de Parkinson e esclerose múltipla (Wang, Bohannon, Kapellusch, Garg, \& Gershon, 2015). Todavia, pesquisas que utilizam o 9-HPT na população atípica, como no TEA, são escassas e não buscaram investigar a lateralidade (Huri, Şahin, \& Kayıhan 2016; Lidstone, Miah, Poston, \& Beasley, 2020).

Considerando que a lateralidade é um domínio importante do movimento, sendo parte integrante do desenvolvimento global da criança, e que há uma precariedade de trabalhos na literatura que buscaram relacionar a lateralidade e a destreza manual em crianças com TEA, este estudo justifica-se pela necessidade de investigaçóes na área do Comportamento Motor com indivíduos com TEA, principalmente no que se refere à observação e à descrição da lateralidade. Dessa forma, o objetivo do presente estudo foi analisar a lateralidade e a destreza manual de crianças com TEA.

\section{Método}

Nesta seção, abordamos os participantes da pesquisa, os instrumentos e a tarefa utilizados com os sujeitos, os procedimentos e os caminhos para a análise dos dados.

\subsection{Participantes}

Participaram inicialmente deste estudo 11 crianças, porém três delas foram excluídas da análise final, pois não conseguiram realizar a tarefa motora. Assim sendo, a amostra final foi

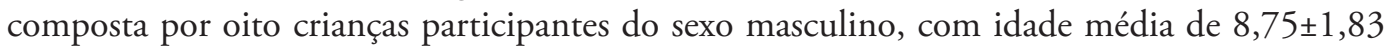
anos e diagnosticadas com TEA com base no DSM-V. A caracterização da amostra pode ser observada na Quadro 1.

Os pais ou responsáveis autorizaram a participação de seus filhos mediante a assinatura do Termo de Consentimento Livre e Esclarecido (TCLE) e as crianças, mediante a assinatura do Termo de Assentimento Livre e Esclarecido (TALE) concordando com a participação voluntária na pesquisa. Como a Instituição onde foi realizada a coleta de dados possuía um maior número de alunos com TEA do sexo masculino, o estudo limitou-se a essa amostra. A prevalência do TEA encontrada no sexo masculino é corroborada pela literatura (Fombonne, 2009).

\subsection{INSTRUMENTOS E TAREFA}

Foi elaborada uma anamnese para a caracterização da amostra, contendo as seguintes questóes: a) Nome; b) Idade; c) Sexo; d) Ano escolar; e) Faz uso de medicamento, se sim, qual; f) Apresenta alguma característica marcante; g) Pratica alguma atividade de lazer; e h) Já teve alguma lesão nas mãos (Quadro 1). Para a determinação do índice de lateralidade, foi solicitado 
aos pais/responsáveis que respondessem ao Inventário de Dominância Lateral de Edimburgo (Oldfield, 1971), considerando a preferência manual dos filhos.

\begin{tabular}{|c|c|c|c|c|c|c|c|}
\hline $\begin{array}{l}\text { Partici- } \\
\text { pantes }\end{array}$ & $\begin{array}{l}\text { Idade } \\
\text { (anos) }\end{array}$ & $\begin{array}{c}\text { Idade } \\
\text { Diagnóstico } \\
\text { (anos) }\end{array}$ & $\begin{array}{c}\text { Ano } \\
\text { Esco- } \\
\text { lar }\end{array}$ & $\begin{array}{c}\text { Uso de } \\
\text { medicamentos }\end{array}$ & $\begin{array}{c}\text { Pratica } \\
\text { atividade* }\end{array}$ & $\begin{array}{c}\text { Lesão } \\
\text { nas mãos }\end{array}$ & Característica marcante \\
\hline 1 & 6 & 4 & $1^{\circ}$ & Não & Sim & Não & $\begin{array}{c}\text { Pisada equinada, } \\
\text { Comportamento opositor }\end{array}$ \\
\hline 2 & 7 & 3 & $2^{\circ}$ & Homeopatia & Sim & Não & $* *$ \\
\hline 3 & 8 & 2 & $3^{\circ}$ & Risperidona & Sim & Não & $* *$ \\
\hline 4 & 8 & 3 & $3^{\circ}$ & Não & Sim & Năo & Ecolalia \\
\hline 5 & 9 & 3 & $4^{\circ}$ & $\begin{array}{l}\text { Risperidona/ } \\
\text { Carbonazepina }\end{array}$ & Sim & Não & Comunicativo \\
\hline 6 & 10 & 4 & $5^{\circ}$ & $\begin{array}{l}\text { Risperidona/ } \\
\text { Ritalina }\end{array}$ & Sim & Não & Hiperativo \\
\hline 7 & 11 & 3 & $6^{\circ}$ & $\begin{array}{l}\text { Ritalina/ } \\
\text { Imprimina }\end{array}$ & Sim & Não & Hiperativo \\
\hline 8 & 11 & 2 & $6^{\circ}$ & $\begin{array}{c}\text { Risperidona/ } \\
\text { Depakene/ } \\
\text { Melleril }\end{array}$ & Sim & Não & $\begin{array}{c}\text { Musicalizado } \\
\text { Hiperativo }\end{array}$ \\
\hline
\end{tabular}

Quadro 1. Caracterização da amostra.

Fonte: Elaborado pelos autores.

* As práticas esportivas realizadas pela amostra são natação e futsal.

** Para os indivíduos 2 e 3, os pais/responsáveis não relataram características marcantes ou/e não souberam responder.

O instrumento utilizado para a tarefa de destreza manual foi o 9-HPT. Essa tarefa é comumente utilizada por terapeutas ocupacionais como uma medida rápida para avaliar a destreza manual por tratar-se de um instrumento preciso e de baixo custo. O 9-HPT consiste em duas placas quadradas e nove pinos de madeira (Figura 1). Uma das placas contém o recipiente para os pinos; e a outra, nove buracos nos quais os pinos se encaixam. A tarefa é realizada em duas condiçóes a serem executadas o mais rápido possível. A primeira condição da tarefa consiste em colocar os pinos em cada buraco, um de cada vez. A segunda condição consiste em retirar os pinos e reposicioná-los no recipiente de origem. Tanto a condição colocar quanto a condição retirar não tiveram uma ordem prescrita; assim, o participante poderia colocar e retirar os pinos da direita para esquerda, de cima para baixo e vice-versa. Para cronometrar o tempo de movimento na tarefa, foi utilizado um cronômetro do celular. A tarefa foi realizada com ambas as mãos. A ordem de início da tarefa foi contrabalançada entre os participantes para eliminar qualquer efeito que a ordem de execução pudesse ter sobre o desempenho motor. 


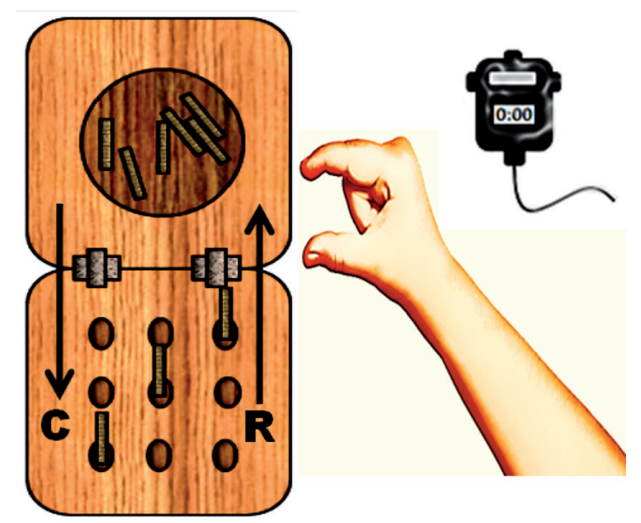

Figura 1. 9-Hole Peg Test.

Fonte: Elaborada pelos autores.

Legenda: C - Colocar; R - Retirar.

\subsection{Procedimentos}

As coletas de dados foram realizadas com os alunos de uma Instituição de ensino pública da cidade de Belo Horizonte, Minas Gerais, mediante autorização da coordenação para a realização do estudo. Em seguida, demos início à coleta de dados. Os pais ou responsáveis assinaram o TCLE, atestando estar de acordo com a participação voluntária do filho no estudo, e os participantes assinaram o TALE. Inicialmente, a coleta de dados consistiu em explicar aos pais de forma detalhada o Inventário de Dominância Lateral de Edimburgo e foi solicitado que os pais respondessem ao questionário. Em seguida, os pais ou responsáveis responderam à anamnese para a caracterização da amostra.

Para a tarefa de destreza manual, o participante foi orientado a sentar-se confortavelmente, com a linha média do corpo alinhada ao 9-HPT que estava em uma mesa. Com a ajuda das professoras do Atendimento Educacional Especializado (AEE) para a aplicação da tarefa de destreza manual, todos os participantes receberam instruçôes verbais padronizadas e uma demonstração da tarefa a ser realizada. No decorrer das instruçóes, foi enfatizado que para a realização da tarefa fosse utilizada apenas à mão designada durante a tentativa. Não foi permitido reposicionar o pino utilizando o corpo, o tabuleiro ou a mesa para ajudar. Se o participante pegasse mais de um pino por vez, mudasse de mão ou utilizasse as duas mãos, posicionasse o pino no corpo, no tabuleiro ou na mesa, era alertado imediatamente sobre a meta da tarefa e eram relembradas as instruçóes iniciais.

A tarefa padrão consiste em uma prática para ambientação e, posteriormente, duas tentativas para cada mão. Entretanto, foram encontradas dificuldades para que os participantes realizassem mais de uma tentativa com cada mão, pois se demonstravam inquietos, impacientes, resistentes e com dificuldade de engajamento na tarefa. Assim, não foi possível realizar a ambientaçấo de todos os participantes. Dessa forma, foi cronometrada apenas uma tentativa com cada uma das mãos.

O tempo de movimento compreendeu o intervalo de tempo entre o momento em que o participante pegou o primeiro pino e posicionou o último pino (Mathiowetz, Weber, 
Kashman, \& Volland, 1985). O tempo não foi corrido durante toda a tarefa, pois foi preciso parar entre as condiçốes colocar e retirar dos pinos, diferentemente do protocolo padrão da tarefa. Essas adaptaçôes foram necessárias por tratar-se de uma amostra que possui um distúrbio complexo com variados graus de severidade. Levando em consideração que a maioria das crianças autistas apresentam dificuldades para lidar com sequências complexas de instruçóes, é necessário que as sequências sejam decompostas em unidades menores (Bosa, 2006).

\subsection{ANÁlISE DOS DADOS}

Foi verificado o princípio de normalidade dos dados por meio do teste de ShapiroWilk ( $p>0,05)$. Para comparar o desempenho das mãos, assim como também o tempo total de execuçáo foram realizados testes $t$ pareado. Foi calculado o tempo total das máos que corresponde à soma dos valores da mão para as condiçóes de colocar e de retirar os pinos. Os erros de execução foram registrados de forma binária, ou seja, apresentou ou não apresentou erro durante a execução do movimento. $\mathrm{O}$ valor de significância adotado foi de $\alpha \leq 0,05$. Para calcular o quociente de lateralidade foi utilizada a seguinte equação: QL= (D-E/D+E) X 100 (Teixeira \& Paroli, 2000), em que $\mathrm{QL}=$ quociente de lateralidade, $\mathrm{D}=$ valores para mão direita, $\mathrm{E}=$ valores para máo esquerda. Os erros de execução foram analisados de forma descritiva.

\section{Resultados}

Nesta seção, tratamos, inicialmente, dos resultados relacionados à preferência lateral; em seguida, sobre a destreza motora; e por, fim, sobre os erros na execução.

\subsection{PreferêNCIA Lateral}

A preferência lateral foi calculada a partir das respostas do Inventário de Dominância Lateral de Edimburgo (Oldfield, 1971). Os resultados podem ser observados na Tabela 1. A partir dos resultados, notamos que a amostra em sua totalidade apresentou preferência manual destra e possui um índice de lateralidade médio de 83,68 pontos.

\begin{tabular}{cc}
\hline Participantes & Quociente de Lateralidade (\%) \\
\hline $\mathbf{1}$ & 64 \\
$\mathbf{2}$ & 100 \\
$\mathbf{3}$ & 100 \\
$\mathbf{4}$ & 60 \\
$\mathbf{5}$ & 100 \\
$\mathbf{6}$ & 63,63 \\
$\mathbf{7}$ & 100 \\
$\mathbf{8}$ & 81,81 \\
Média (DP) & $83,68( \pm 18,59)$ \\
\hline
\end{tabular}

Tabela 1. Resultados do Inventário de Dominância Lateral de Edimburgo. Fonte: Elaborada pelos autores. 


\subsection{Destreza MOTORA}

Para avaliar a destreza motora, foi analisado o desempenho no 9-HPT por meio dos tempos gastos durante as condiçóes de colocar e retirar os pinos, assim como também o tempo total de cada mão (soma dos tempos das condiçóes colocar e retirar). $\mathrm{O}$ teste t detectou diferenças significativas entre as mãos na condição de colocar os pinos $[\mathrm{t}(\mathrm{df}=14)=-2,55, \mathrm{p}=0,03]$ (Figura 2). A mão preferida apresentou melhor desempenho, tempo médio de 20,03 segundos, que a mão não preferida, 24,91 segundos. Já para a condição de retirar os pinos, o teste t não detectou diferença significante entre as mãos $[\mathrm{t}(\mathrm{df}=14)=-1,44, \mathrm{p}=0,19]$ (Figura 3). A mão preferida apresentou um tempo médio de 9,91 segundos; enquanto a mão não preferida, 12, 06 segundos.

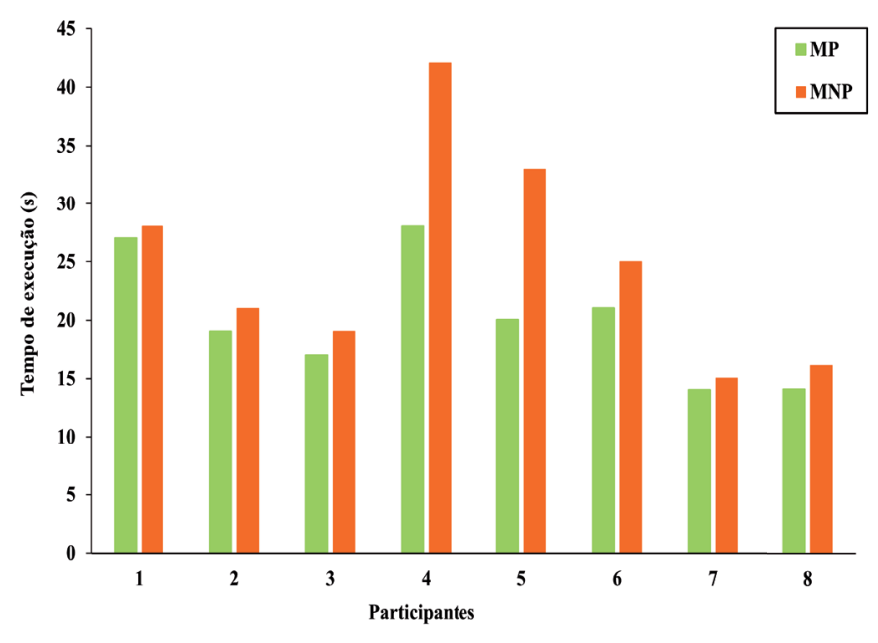

Figura 2. Tempo de execução durante a tarefa de colocar os pinos.

Fonte: Elaborada pelos autores.

* $\mathbf{M P}=$ mão preferida; $\mathbf{M N P}$ = mão não preferida. 


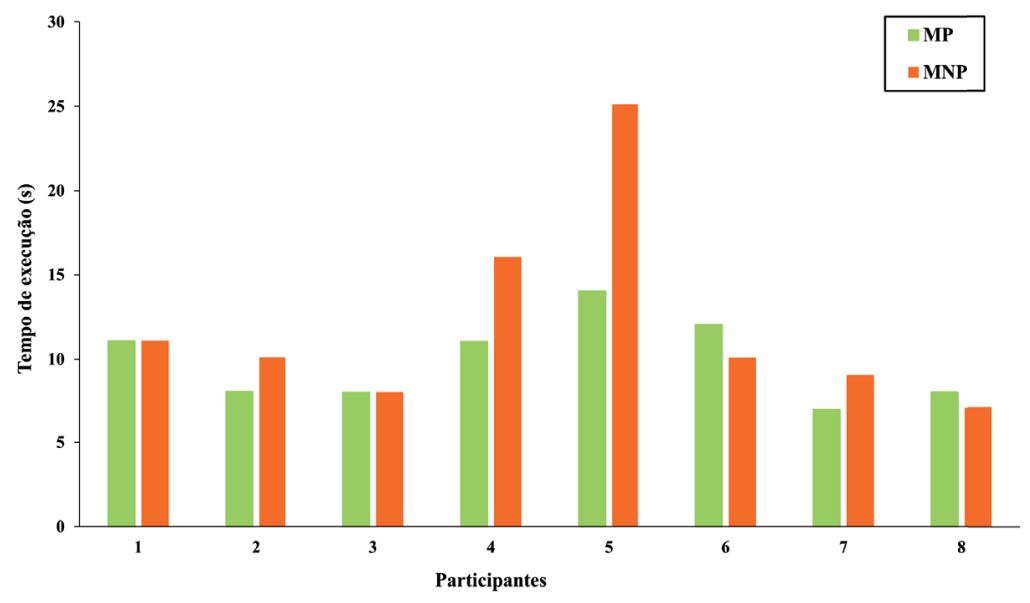

Figura 3. Tempo de execução durante a tarefa de retirar os pinos.

Fonte: Elaborada pelos autores.

* $\mathbf{M P}$ = mão preferida; $\mathbf{M N P}$ = mão não preferida.

Para a análise do tempo total de execução das mãos, o teste t apresentou uma diferença marginal no desempenho $[\mathrm{t}(\mathrm{df}=14)=-2,18, \mathrm{p}=0,06]$ (Figura 4). A mão preferida apresentou melhor desempenho quando comparado à mão não preferida.

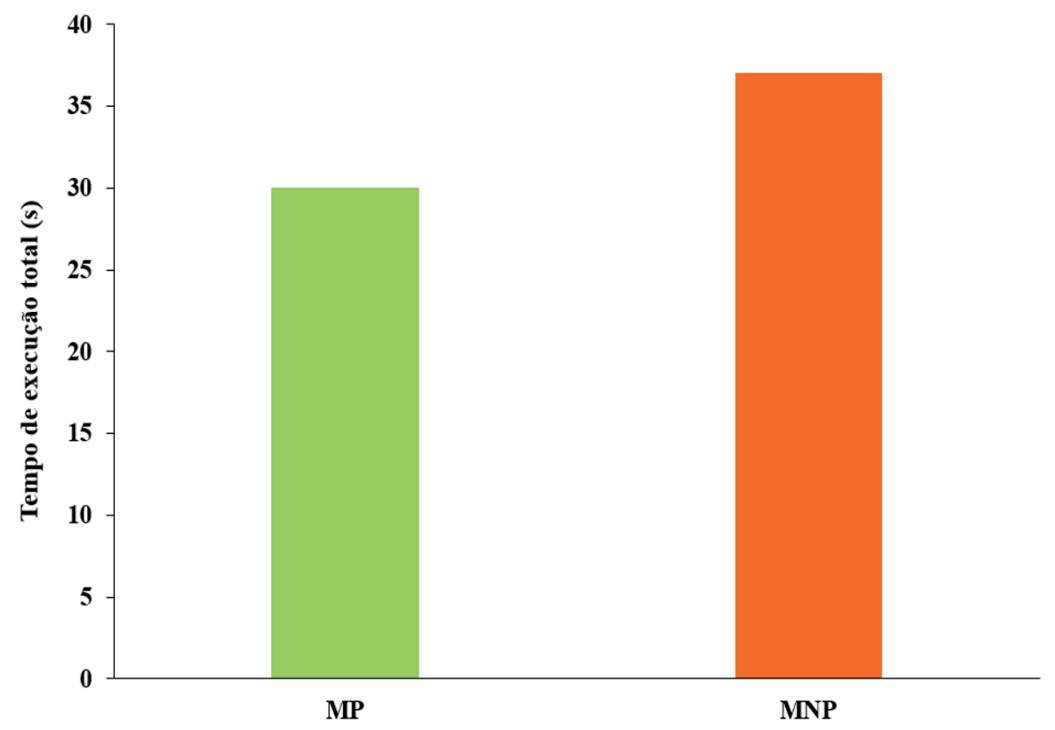

Figura 4. Tempo total de execução das mãos.

Fonte: Elaborada pelos autores.

${ }^{*} \mathbf{M P}$ = mão preferida; $\mathbf{M N P}$ = mão não preferida. 


\subsection{ERros NA EXeCuÇáo}

Para melhor analisar a destreza manual na tarefa aplicada, foram analisados os erros recorrentemente cometidos pelos participantes. Os erros observados foram: a) utilizou outra mão; b) pegar mais de um pino; e c) deixar cair o pino. Os erros foram computados de modo binário, ou seja, sim ou não, para contabilizar quantos participantes erraram e quais os erros cometidos.

Utilizou outra máo: foi considerado o uso da outra mão quando o participante utilizou a mão que não estava destinada a realizar a tarefa para auxiliar ou pegou algum pino com a mão que não estava sendo avaliada no momento. Foram contabilizados os erros das condiçôes colocar e retirar os pinos, separadamente. De forma geral, a mão preferida apresentou maior número de erros comparada à mão não preferida; além disso, a condição de retirar mostrou o maior número de erros quando comparado à condição de colocar (Figura 5).

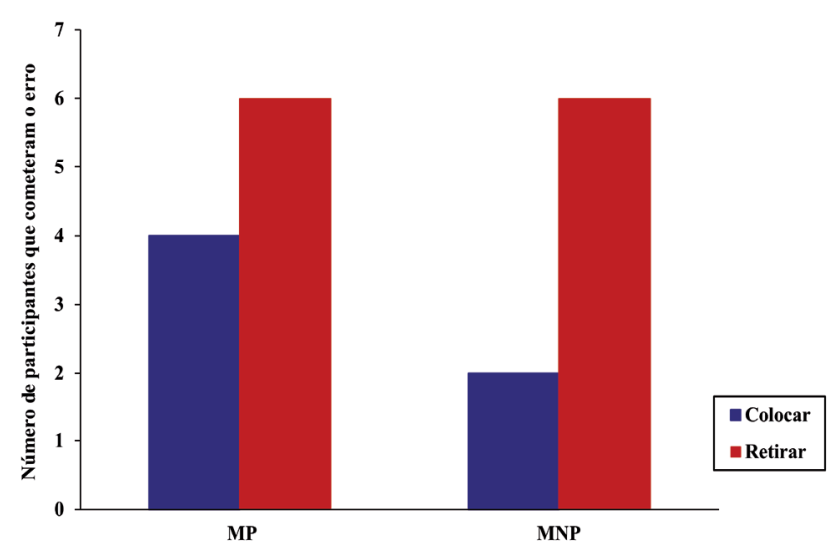

Figura 5. Erro de execução utilizou outra mão.

Fonte: Elaborada pelos autores.

${ }^{*} \mathbf{M P}$ = mão preferida; $\mathbf{M N P}$ = mão não preferida.

Pegou mais de um pino: foi considerado pegar mais de um pino quando o participante segurou dois pinos ou mais com a mão que executava a tarefa. Foram contabilizados os erros das condiçóes colocar e retirar os pinos, separadamente. Ao analisarmos os dados, notamos que de forma geral havia um maior número de erros cometidos pela mão não preferida, comparada à mão preferida. Além disso, a condição de retirar mostrou o maior número de erros quando comparado à condição de colocar (Figura 6). 


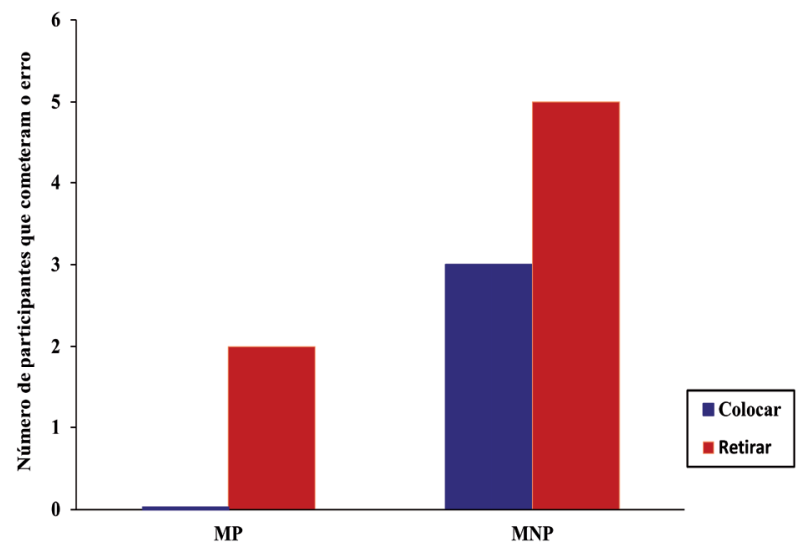

Figura 6. Erro de execução pegar mais de um pino.

Fonte: Elaborada pelos autores.

${ }^{*} \mathbf{M P}$ = mão preferida; $\mathbf{M N P}$ = mão não preferida.

Deixou cair um pino: foi considerado deixar cair um pino quando o participante perdeu o controle do pino e consequentemente o deixou cair. Foram contabilizados os erros das condiçóes colocar e retirar os pinos, separadamente. Foi observado que, diferentemente do erro "usar outra mão" e "pegar mais de um pino", o maior número de erros ocorreu na condição colocar os pinos. De forma geral, a mão não preferida apresentou maior número de erros comparada à mão preferida (Figura 7).

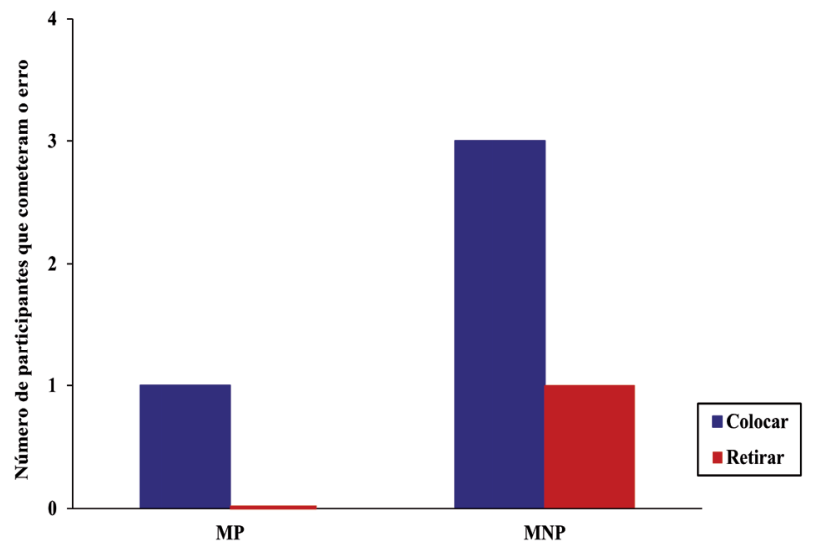

Figura 7. Média do erro de execução deixar cair um pino.

Fonte: Elaborada pelos autores.

* $\mathbf{M P}$ = mão preferida; $\mathbf{M N P}$ = mão não preferida. 


\section{Discussão}

Considerando a lateralidade como um domínio importante do comportamento humano, este estudo teve como objetivo analisar a lateralidade e a destreza manual de crianças com TEA. Os resultados indicaram uma média de 83,68\% para o índice de lateralidade, confirmando a direçáo da lateralidade para a mão direita para a amostra do estudo. Esse resultado corrobora com a distribuição majoritária de destros na população (Annett, 1967). Ao observar o desempenho na tarefa motora de destreza manual, foi registrado um desempenho superior no tempo total de execuçáo para os movimentos realizados com a mão preferida.

Apesar da maioria dos participantes apresentarem um desempenho superior com a mão preferida, alguns participantes não mostraram esse padrão entre as diferentes condiçóes da tarefa. De acordo com McManus, Murray, Doyle e Baron-Cohen (1992) e Paquet et al. (2017), crianças autistas mostram discordância entre a mão preferida e a mais habilidosa. A escolha entre mãos começa a ser determinada por volta de dois anos de idade, mas não é totalmente estabelecida até os três ou quatro anos de idade (Gesell \& Ames, 1947). Contudo, há uma tendência dessa lateralização fortalecer-se ao longo da vida.

Em relação à tarefa motora, foi observada diferença significativa no desempenho das mãos na condição de colocar os pinos, assim como um indicativo no tempo total de execução. Consideramos que a mão preferida apresentou melhor desempenho, ainda que marginal $(\mathrm{p}=0,06)$, em relaçáo ao desempenho do tempo total de execução da mão não preferida. Em ambos, a mão preferida apresentou um desempenho superior. De forma descritiva, observamos, também, que a máo preferida apresentou menor número de erros totais comparado à mão não preferida. Esses resultados mostram que a mão preferida parece apresentar melhor destreza manual. A destreza motora é entendida como a capacidade de produzir os resultados previstos com o máximo de êxito e com o mínimo de custo de tempo, energia ou ambas as coisas (Knapp, 1963). Nos autistas, é frequente encontrar défcits no desempenho motor como o controle da força, a orientação espaço-temporal, equilíbrio e da própria lateralidade (Vasconcelos, Rodrigues, Barreiros, \& Jacobsohn, 2009), que sáo capacidades necessárias para a destreza manual.

Foram analisados os erros: "utilizou a outra mâo", "pegou mais de um pino" e "deixou cair um pino". Houve uma maior incidência de erros cometidos pela mão não preferida, sugerindo uma possível explicação para a diferença significativa no tempo total da tarefa entre as mãos, cuja mão preferida obteve menor tempo de execução. Outro ponto a ser discutido é que a maior parte dos erros ocorreu na condição de retirar os pinos, uma possível explicação para tal resultado se dá por meio da Lei de Fitts, de 1959: quanto maior a velocidade menor a precisão. Assim, devido à tarefa exigir menor atençáo por oferecer menor dificuldade de realização, o tempo gasto na tarefa foi menor, mas a possibilidade do erro se torna maior, comparado à condição de colocar os pinos. Seguindo essa lógica, a diferença significativa no tempo total da tarefa e da não diferença no tempo do retirar pode ser explicada também pela dificuldade da tarefa. Colocar os pinos requer maior destreza e atenção na execução, resultando em uma maior duração da tarefa e ressaltando as diferenças entre as mãos. Entretanto, retirar os pinos parece exigir menos das capacidades motoras e cognitivas, resultado, assim, em discretas diferenças no desempenho das mãos. 
É importante relatarmos um fator que pode ter interferido no estudo, a motivação. Apesar de não ter sido o foco do presente estudo e também não ter sido diretamente avaliada com instrumentos adequados, algumas informaçóes podem complementar o entendimento dos resultados encontrados. Após a demonstração da tarefa, quando foi solicitado para que os participantes a realizassem, alguns nấo quiseram, sendo necessário insistir para que a tarefa fosse realizada. Além disso, alguns participantes mostraram resistência para realizar a tentativa com a mão contralateral da mão que iniciou a tarefa. Segundo o estudo de Cornish e McManus (1996), a motivação pode ser uma variável importante para o desempenho motor em tarefas que avaliam o tempo de movimento, pois evidências apontam que o baixo desempenho motor pode estar relacionado ao baixo engajamento na tarefa, já que tarefas pouco motivantes podem não incentivar o foco de atenção para uma execução rápida.

O 9-HPT apresenta evidências de um bom grau de confiabilidade (Smith, Hong, \& Presson, 2000) para todas as idades dentro de sua amostra normativa. No entanto, não há pesquisas publicadas sobre a confiabilidade dessa tarefa motora com crianças com TEA. Além disso, pesquisas em relação ao tema são escassas, o que dificulta comparaçóes dos nossos resultados com os de outros autores, para verificar se os scores obtidos estáo dentro do padrăo esperado para essa população. Por exemplo, o estudo de Smith, Hong e Presson (2000) comparou o desempenho de crianças que frequentavam a Educação Especial com crianças da educaçáo regular, os resultados do estudo mostraram que as crianças da Educaçáo Especial apresentaram um tempo maior para completar a tarefa; além disso, para ambos os grupos, a mão preferida apresentou melhor desempenho. Entretanto, o método utilizado por Smith, Hong e Presson (2000) foi distinto do utilizado neste estudo e, na amostra de crianças matriculadas na Educação Especial, não há uma caracterização desses participantes, fato que dificulta fazer comparações com nossos resultados. O que se pode comparar são os tempos médios de crianças com desenvolvimento típico com nossa amostra. Essa comparação permite identificar que as crianças com TEA parecem ser mais lentas na execuçáo da tarefa (Smith, Hong, \& Presson, 2000). Ademais, corrobora com outros estudos que aplicaram testes distintos e que mostram o pior desempenho de autistas em uma habilidade motara fina em comparação a crianças típicas (Leal, 2011; Provost, Heimerl, \& Lopez, 2007).

Uma vez que a lateralidade pode atuar como um marcador comportamental, esses resultados podem orientar a prática interventiva dos profissionais da saúde como professores, fisioterapeutas e terapeutas ocupacionais. A partir do entendimento da relação entre a lateralidade e o desenvolvimento da aquisição da escrita, destreza manual e linguagem, os profissionais podem adotar estratégias de intervençáo motora bilateral, desenvolvendo não só o domínio motor da lateralidade, mas também auditivo e visual. Além disso, a partir desses resultados, podemos atentar para a utilizaçâo de testes que contemplem o lado lúdico das tarefas motoras com intuito de motivar a sua execuçáo. Diante das diferentes formas de apresentaçấo do Transtorno, torna-se desafiador estabelecermos métodos de análise do movimento que orientem a intervenção, mas é possível orientar sobre aspectos gerais dessa prática.

Devido às características do grupo avaliado associado às questóes relacionadas à motivação para a prática, não foi possível realizar a tarefa mais de uma vez. $\mathrm{O}$ recomendado é que sejam realizadas ao menos três tentativas da tarefa, assim como Davis, Fenlon, Proctor e Watson (1997) demonstraram em seu estudo, apontando que a média das três tentativas 
produz maior confiabilidade dos resultados. Portanto, esse aspecto pode ser considerado uma limitação do estudo. Sugerimos, assim, que estudos futuros busquem estratégias para garantir o engajamento das crianças na tentativa de realizar a tarefa conforme recomendado. Além disso, durante a aplicação da anamnese, a delimitação de características das crianças pode auxiliar na compreensão dos resultados. Neste estudo, os pais pontuaram como características marcantes aspectos distintos, como emocionais, físicos e comportamentais. Essa delimitação pode ampliar a compreensão do comportamento motor desses indivíduos. Assim, considerando o espectro de características presentes no TEA, os resultados apresentados são considerados exploratórios e suas generalizaçóes devem ser realizadas de modo cauteloso.

Outra aspecto a ser considerado como limitação do estudo se refere ao tamanho da amostra. Apesar de se tratar de uma população específica, estudos futuros podem realizar a análise com uma amostra mais ampla e em diferentes faixas etárias e sexos para aumentar o poder de generalização dos resultados. A presente amostra foi composta por crianças de 6 a 11 anos, praticantes de atividades físicas, estudantes do ensino regular público, com uso sistemático de medicação. Essas características devem ser consideradas. Em suma, as habilidades motoras são relatadas como um importante preditor de desempenho nas atividades da vida diária, como exemplo a caligrafia e função escolar (Kopp, Beckug, \& Gillberg, 2010). Deve ser levado, portanto, em consideração, sua relevância para o desenvolvimento integral da criança com TEA.

\section{Conclusấo}

Este estudo mostrou que os autistas participantes do estudo parecem apresentar lateralidade destra e também melhor desempenho com a mão preferida em uma tarefa de destreza manual. Observamos que, na condiçãao de colocar os pinos que exigiu maior atenção e destreza, a diferença no desempenho das mãos foi significativa. Na condição de retirar os pinos, que exige menor demanda atencional e de destreza manual, o desempenho das mãos não foi significante; além disso, nesse momento, foram observados maiores erros na execução. Sendo a lateralidade um domínio importante do desenvolvimento global do indivíduo, é importante identificar défcits nessa dimensão para orientar a intervenção motora com o intuito de diminuir as dificuldades motoras que as crianças com TEA apresentam e, assim, melhorar a qualidade de vida, sensação de bem-estar e autonomia dessa população.

\section{REFERÊNCIAS}

American Psychiatric Association (2013). Diagnostic and statistical manual of mental disorders (DSM- $5^{\circ}$ ). American Psychiatric Pub.

Annett, M. (1967). The binomial distribution of right, mixed and left handedness. The Quarterly journal of experimental psychology, 19(4), 327-333.

Aram, D., Best, C., Betz, J., Brumback, R., Bryden, M., Carlson, D. ... Whitaker, H. (1988). Brain Lateralization in Children: Developmental implications. New York: The Guilfor Press.

Bosa, C. A. (2006). Autismo: intervençóes psicoeducacionais. Brazilian Journal of Psychiatry, 28, s47-s53. 
Caetano, D. (1993). Classificação de transtornos mentais e de comportamento da CID-10: descriçôes clínicas e diretrizes diagnósticas. In Classificação de Transtornos Mentais e de Comportamento da CID-10: Descriçôes clínicas e diretrizes diagnósticas. Porto Alegre: Artes Médicas.

Catelli, C. L. R. Q., D’Antino, M. E. F., \& Assis, S. M. B. (2016). Aspectos motores em indivíduos com transtornos do espectro autista: revisão de literatura. Cadernos de Pós-Graduação em Distúrbios do Desenvolvimento, 16(1), 56-65.

Chukoskie, L., Townsend, J., \& Westerfield, M. (2013). Motor skill in autism spectrum disorders: a subcortical view. International Review of Neurobiology, 113, 207-249. DOI: https://doi. org/10.1016/b978-0-12-418700-9.00007-1

Cornish, K. M., \& McManus, I. C. (1996). Hand preference and hand skill in children with autism. Journal of Autism and Developmental Disorders, 26(6), 597-609.

Davis, J., Fenlon, P., Proctor, T., \& Watson, W. (1997). The Nine Hole Peg Test: A comparison study. Unpublished master's thesis, Kirksville College of Osteopathic Medicine, Southwest Center for OME and HS, Mesa, AZ.

Floris, D. L., \& Henrietta, H. (2018). Atypical structural and functional motor networks in autismo. Progress in Brain Research, 238, 207-248.

Fombonne, E. (2009). Epidemiology of pervasive developmental disorders. Pediatric research, 65(6), 591-598.

Forrester, G. S., Ruth, P., Thomas, M. S. C., \& Mareschal, D. (2014). Handedness as a marker of cerebral lateralization in children with and without autism. Behavioural Brain Research, 268(15), $14-21$.

Gesell, A., \& Ames, L. B. (1947). The development of handedness. The Pedagogical Seminary and Journal of Genetic Psychology, 70(2), 155-175.

Günal, A., Bumin, G., \& Huri, M. (2019). The Effects of Motor and Cognitive Impairments on Daily Living Activities and Quality of Life in Children with Autism. Journal of Occupational Therapy, Schools, \& Early Intervention, 12(4), 444-454. DOI: https://doi.org/10.1080/19411243.2019.16 04286

Hauck, J. A., \& Dewey, D. (2001). Hand preference and motor functioning in children with autism. Journal of Autism and Developmental Disorders, 31(3), 265-277.

Huri, M., Şahin, S., \& Kayıhan, H. (2016). Investigation of Hand Function Among Children Diagnosed with Autism Spectrum Disorder with Upper Extremity Trauma History. Ulus Travma Acil Cerrahi Derg, 22(6), 559-565.

Knapp, B. (1963). Skill in sport: the attainment of proficiency. London: Routledge \& K. Paul.

Kopp, S., Beckung, E., \& Gillberg, C. (2010). Developmental coordination disorder and other motor control problems in girls with autism spectrum disorder and/or attention-deficit/hyperactivity disorder. Research in developmental disabilities, 31(2), 350-361.

Kummer, A., \& Teixeira, A. L. (2007). O autismo como um transtorno do movimento. Revista Brasileira de Neurologia, 43(2), 20-25.

Leal, S. F. M. (2011). Autismo e Lateralidade-estudo da Preferência Manual através do Card-reaching Test. Dissertação de Mestrado, Faculdade de Desporto, Universidade do Porto, Porto, Portugal. 
Lidstone, D. E., Miah, Z. F., Poston, B., \& Beasley, J. F. (2020). Manual dexterity in children with autism spectrum disorder: A cross-syndrome approach. Research in Autism Spectrum Disorders, $73,1-8$.

Lloyd, M., MacDonald, M., \& Lord, C. (2013). Motor skills of toddlers with autism spectrum disorders. Autism, 17(2), 133-146.

Mathiowetz, V., Weber, K., Kashman, N., \& Volland, G. (1985). Adult norms for the nine hole peg test of finger dexterity. The Occupational Therapy Journal of Research, 5(1), 24-38.

McManus, I. C., Murray, B., Doyle, K., \& Baron-Cohen, S. (1992). Handedness in childhood autism shows a dissociation of skill and preference. Cortex, 28(3), 373-381.

Molfese, D. L., \& Segalowitz, S. J. (1988). Brain Lateralization in children: Developmental implications. New York: Guilford Press.

Oldfield, R. C. (1971). The assessment and analysis of handedness: The Edinburgh inventory. Neuropsychologia, 9(1), 97-113.

Paquet, A., Golse, B., Girard, M., Olliac, B., \& Vaivre-Douret, L. (2017). Laterality and Lateralization in Autism Spectrum Disorder, Using a Standardized Neuro-Psychomotor Assessment Developmental. Neuropsychology, 42(1), 39-54.

Pereira, A., Riesgo, R. S., \& Wagner, M. B. (2008). Autismo infantil: tradução e validação da Childhood Autism Rating Scale para uso no Brasil. Jornal de Pediatria, 84(6), 487-494.

Poole, J. L., Burtner, P. A., Torres, A. T., McMullen, C. K., Markham, A., Marcum, M. L., ... Qualls, C. (2005). Measuring dexterity in children using the Nine-hole Peg Test, J Hand Ther, 18(3), 348351 .

Preslar, J., Kushner, H. I., Marino, L., \& Pearce, B. (2013). Autism, lateralisation, and handedness: A review of the literature and meta-analysis. Laterality: Asymmetries of Body, Brain and Cognition, 19(1), 64-95.

Provost, B. B. R., Heimerl, S., \& Lopez, B. R. (2007). Levels of gross and fine motor development in young children with autism spectrum disorder. Physical \& Occupational Therapy in Pediatrics, 27(3), 21-36.

Rodriguez, A., Kaakinen, M., Moilanen, I., Taanila, A., McGough, J. J., Loo, S., \& Järvelin, M. R. (2010). Mixed-handedness is linked to mental health problems in children and adolescents. Pediatrics, 125(2), 340-348.

Romero, E. (1988). Lateralidade e rendimento escolar. Revista Sprint, 6(1), 23-26.

Rosa Neto, F. (2002). Manual de Avaliação Física. Porto Alegre: Artmed.

Rutter, M., \& Thapar, A. (2014). Genetics of autism spectrum disorders. In F. R. Volkmar, R. Paul, S. J. Rogers, \& K. A. Pelphrey (Eds.), Handbook of Autism and Pervasive Developmental Disorders ( $4^{a}$ ed., pp. 411-423). Hoboken: John Wiley \& Sons.

Smith, Y. A., Hong, E., \& Presson, C. (2000). Normative and validation studies of the Nine-hole Peg Test with children. Perceptual and motor skills, 90(3), 823-843.

Soares, A. M., \& Cavalcante Neto, J. L. (2015). Avaliação do comportamento motor em crianças com transtorno do espectro do autismo: uma revisão sistemática. Revista Brasileira de Educação Especial, 21(3), 445-458. 
Teixeira, L. A., \& Paroli, R. (2000). Assimetrias laterais em ações motoras: preferência versus desempenho. Motriz, Journal of Physical Education, 6(1), 1-8.

Thompson, A., Murphy, D., Dell'Acqua, F., Ecker, C., McAlonan, G., Howells, H., ... Catani, M. (2017). Impaired communication between the motor and somatosensory homunculus is associated with poor manual dexterity in autism spectrum disorder. Biological Psychiatry, 81(3), 211-219.

Vasconcelos, O., Rodrigues, P., Barreiros, J., \& Jacobsohn, L. (2009). Laterality, developmental coordination disorders and posture. In L. Paulo Rodrigues, J. Barreiros, \& O. Vasconcelos (Eds.), Estudos em desenvolvimento motor da criança II. (pp. 19-26). Leiria: ESE-IPVC Ediçóes

Velasco, C. G. (1996). Brincar: o despertar psicomotor. Rio de Janeiro: Sprint.

Vitorino, M. D. S. (2014). Perfil funcional e sensorial de crianças com autismo: implicaçóes para a terapia ocupacional (Trabalho de conclusão de curso), Departamento de Terapia Ocupacional, Universidade Federal da Paraíba, João Pessoa, Paraíba, Brazil.

Wang, Y.-C., Bohannon, R. W., Kapellusch, J. Garg, A., \& Gershon, R. C. (2015). Dexterity as measured with the 9-Hole Peg Test (9-HPT) across the age span. Journal of Hand Therapy, 28(1), 53-60. https://doi.org/10.1016/j.jht.2014.09.002

Zilbovicius, M., Meresse, I., \& Boddaert, N. (2006). Autismo: neuroimagem. Revista Brasileira Psiquiatria, 28(1), 521-528.

Recebido em: 29/04/2020

Reformulado em: 25/06/202

Aprovado em: 06/07/202 
FERNANDES, L.A. et al. 